\title{
Modified Self-Organizing Feature Map Neural Network with Semi-supervision for Change Detection in Remotely Sensed Images
}

\author{
Susmita Ghosh and Moumita Roy \\ Department of Computer Science and Engineering, Jadavpur University, \\ Kolkata 700032, India
}

\begin{abstract}
Problem of change detection of remotely sensed images using insufficient labeled patterns is the main topic of present work. Here, semisupervised learning is integrated with an unsupervised context-sensitive change detection technique based on modified self-organizing feature map (MSOFM) network. In this method, training of the MSOFM is performed iteratively using unlabeled patterns along with a few labeled patterns. A method has been suggested to select unlabeled patterns for training. To check the effectiveness of the proposed methodology, experiments are carried out on two multitemporal remotely sensed images. Results are found to be encouraging.
\end{abstract}

Keywords: Semi-supervised learning, change detection, fuzzy set, selforganizing feature map.

\section{Introduction}

Change detection, a process of finding out changes in a land cover over time by analyzing remotely sensed images of a geographical area captured at different times [9], can be viewed as an image segmentation problem, where two groups of pixels (changed and unchanged) are formed. Between the two traditional approaches (supervised and unsupervised), the applicability of supervised method [1] is less for insufficient ground truth information. Whereas, ground truth is not necessary for unsupervised methods [15. Due to the depletion of labeled patterns, unsupervised techniques seem to be compulsory here. The three consecutive steps used for unsupervised technique are image preprocessing, image comparison and image analysis [9. In preprocessing step, images are made compatible by operations like radiometric and geometric corrections, co-registration etc. Then, image comparison is done pixel by pixel to generate a difference image (DI). A situation may occur where the categorical information of a few labeled patterns can be collected easily by experts. If the number of these labeled patterns is less, then this information may not be sufficient for developing supervised methods. In such a scenario, knowledge of labeled patterns, though very poor, will be unutilized if unsupervised approach is taken. Under this circumstance, semi-supervised approach 2 could be opted instead of unsupervised or supervised one. 
In the present work, a neural network based semi-supervised change detection method is proposed. Here, the idea of semi-supervised learning [2] is incorporated into the existing unsupervised change detection technique based on the MSOFM network 6]. This work is inspired from a work on change detection using semi-supervised multilayer perceptron (MLP) [8]. The MSOFM network [46] has two layers: input and output. For each feature of the input pattern, there is a neuron in the input layer. The output layer is two dimensional and there is a representative neuron for each pixel position of DI. In the present work, change vector analysis (CVA) technique [19] is used for generating DI. The input pattern for a particular pixel position is generated using gray value of the said pixel as well as those of its neighborhood pixels for considering (spatial) contextual information from neighbors. In the present work $2^{\text {nd }}$ order neighborhood is used. A mapping algorithm [46] is used to normalize the feature values of the input pattern in $[0,1]$. Each neuron in the output layer is connected to all the neurons in the input layer. The connection weights of the representative neuron of the labeled pixel position are initialized with the normalized feature values of the corresponding labeled pattern; whereas the weight vector for others is initialized randomly between $[0,1]$. Iterative learning of the MSOFM network is performed by the labeled patterns along with the selected unlabeled patterns until a given convergence criterion is satisfied.

\section{Kohonen's Self-Organizing Feature Map}

In the proposed algorithm, we have used the same MSOFM network as used in 46]. The MSOFM network is motivated from the Kohonen's self-organizing feature maps (SOFM) [7] and has similar network structure as that of the SOFM. SOFM is learned iteratively and generates topological map of input patterns gradually. The input pattern is represented by $\vec{X}$ and $\overrightarrow{W_{j}}$ denotes the synaptic weight vector of $j^{\text {th }}$ output neuron (where $j=1,2, \ldots, z$ and $z$ be the number of neurons in the output layer). The winner neuron $j$ in the output layer is selected as the best-matched neuron for the given input pattern where the similarity measure $\left(\overrightarrow{W_{j}} \cdot \vec{X}\right)$ is maximum. Let, $h(i t r)$ be the topological neighborhood function at iteration $i t r$ that shrinks after each iteration. The weight updating of winning neuron is done as,

$$
\vec{W}(i t r+1)=\vec{W}(i t r)+h(i t r) \eta(i t r)(\vec{X}-\vec{W}(i t r))
$$

where, $\eta(i t r)$ denotes the learning rate in iteration $i t r$ and decreases with increase of $i t r$.

\section{The Proposed Algorithm}

In the present technique, labeled patterns from both the classes are picked up randomly from the ground truth. Let, the $y$-dimensional input pattern of $(m, n)^{t h}$ pixel position of DI is denoted by $\overrightarrow{X_{m n}}=\left[x_{m n, 1}, x_{m n, 2}, \ldots, x_{m n, y}\right]$. The 
$y$-dimensional weight vector between $(m, n)^{t h}$ neuron in the output layer and all the neurons of the input layer is represented by $\overrightarrow{W_{m n}}=\left[w_{m n, 1}, w_{m n, 2}, \ldots, w_{m n, y}\right]$. During training, input patterns $\overrightarrow{X_{m n}}$ are passed to the network one by one. Each time, the dot product $d(m, n)$ between $\overrightarrow{X_{m n}}$ and $\overrightarrow{W_{m n}}$ is calculated as,

$$
d(m, n)=\overrightarrow{X_{m n}} \cdot \overrightarrow{W_{m n}}=\sum_{k=1}^{y} x_{m n, k} \cdot w_{m n, k} .
$$

At the beginning of the training phase, the connection weights of the MSOFM network are updated in the following manner using the labeled patterns only. If the class label of the $(m, n)^{t h}$ pixel position is known, then the weight vector $\overrightarrow{W_{i j}}$ for all neighboring neurons (defined by $\left.h_{m n}().\right)$ of $(m, n)^{t h}$ output neuron is updated using equation (1), iff $(i, j)^{t h}$ input pattern is unlabeled. For the labeled patterns, the weight vector is not updated during the entire learning process. Learning using labeled patterns is continued iteratively until convergence. To check convergence, $O(i t r)$ is calculated at each iteration using,

$$
O(i t r)=\sum_{d(m, n) \geq t} d(m, n),
$$

where, $t$ is a predefined threshold value 6]. The weight updating is preformed until $(O(i t r)-O(i t r-1))<\delta$, where $\delta$ is a small positive quantity. The value of $t$ varies within $[0,1]$. The components of the weight vector $\overrightarrow{W_{m n}}$ are normalized in a way so that the dot product $d(m, n)$ lies between $[0,1]$. After each epoch, the learning rate $\eta(i t r)$ and the size of the topological neighborhood $h(i t r)$ decrease.

After each training step, the soft class labels of unlabeled patterns are calculated by presenting them to the network. Let us consider that there exists two fuzzy sets: one for the changed class and the other for unchanged one. For each $(i, j)^{t h}$ unlabeled pattern, $d(i, j)$ is computed by equation $(2)$; if, $d(i, j) \geq t$, then $(i, j)^{t h}$ pattern is more likely to belong to changed class than unchanged one; else in unchanged class. Let, $\mu(i, j)=\left[\mu_{1}(i, j), \mu_{2}(i, j)\right]$ be the membership value of $(i, j)^{t h}$ unlabeled pattern, where $\mu_{1}(i, j)$ and $\mu_{2}(i, j)$ are the membership values in unchanged class and changed class, respectively. The values can be calculated as,

$$
\left[\mu_{1}(i, j), \mu_{2}(i, j)\right]=\left\{\begin{array}{l}
{[\min (d(i, j), 1-d(i, j)),} \\
\max (d(i, j), 1-d(i, j))] \\
{[\max (d(i, j), 1-d(i, j)),} \\
\min (d(i, j), 1-d(i, j))]
\end{array} \quad\right. \text { Otherwise. }
$$

After that, the target value of the unlabeled pattern is updated by $K$-nearest neighbor technique as it was used in [8]. To search for $K$-nearest neighbors, instead of considering all the patterns, we consider only those which lie within a window around that unlabeled pattern. Let, $M$ be the set of $K$-nearest neighbors of $(i, j)^{t h}$ unlabeled pattern. Now, target value $t(i, j)=\left[t_{1}(i, j), t_{2}(i, j)\right]$ of $(i, j)^{t h}$ unlabeled pattern is estimated as, 


$$
t(i, j)=\left[\frac{\sum_{\overrightarrow{X_{s l}} \in M} \mu_{1}(s, l)}{K}, \frac{\underset{X_{s l} \in M}{\longrightarrow} \mu_{2}(s, l)}{K}\right] .
$$

Then, the unlabeled patterns which are more likely to belong to the changed class, are selected for training the MSOFM network again. The process of training the MSOFM using labeled patterns and the selected unlabeled patterns continued until convergence. The training of network and re-estimation of soft class labels of unlabeled patterns using equations (4) and (5) are continued iteratively (for DI of size $p \times q$ ) by computing the sum of square error, $\xi(N)$, after each $N^{\text {th }}$ training step as,

$$
\xi(N)=\sum_{i=1}^{p} \sum_{j=1}^{q} \sum_{k=1}^{2}\left(\mu_{k}(i, j)-t_{k}(i, j)\right)^{2} .
$$

\section{Results and Analysis}

To evaluate the effectiveness of the proposed method, experiments are carried out on two multitemporal remotely sensed images corresponding to the geographical areas of Mexico and Sardinia island. Similar findings are obtained for both the data sets. For illustration, results of Mexico data sets are presented here. The performance of the proposed technique is compared with those of the existing unsupervised technique using MSOFM [6] and a supervised method using MLP. The performance indices are the number of missed alarms (changed class pixels identified as unchanged ones- $M A$ ), the number of false alarms (unchanged class pixels classified as changed ones- $F A$ ), the number of overall errors $(O E)$ and Kappa measure $(K M)$ [3. The average (Avg.) and standard deviation (written in brackets in the table) values (over 10 simulations) of overall errors and Kappa measure are considered for comparison. The best results (denoted by 'Min' in table) and the worst results (denoted by 'Max' in table) for $M A, F A$ and $O E$, considering all the simulations, are also given in the table.

The data set of Mexico is made of two multispectral images over an area of Mexico 68] taken on April 18, 2000 and May 20, 2002 (Fig. 11). The corresponding difference image and the reference map are shown in Figure 1 The change detection maps corresponding to minimum overall error (over 10 simulations) obtained using optimal threshold with the unsupervised technique and the proposed semi-supervised technique (using $0.1 \%$ patterns from the changed class and $0.1 \%$ from the unchanged class) are depicted in Figure 2

Table 1 presents the comparative results obtained using the unsupervised method, the supervised method and the proposed semi-supervised method. From the table, it is noticed that the proposed method (considering all the cases of percentage of training patterns) outperforms the corresponding unsupervised version. It is also found that the standard deviations obtained for both overall error and Kappa measure using MLP based supervised technique are very high 


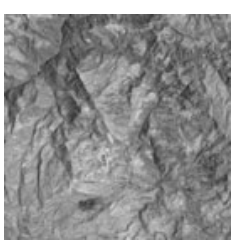

(a)

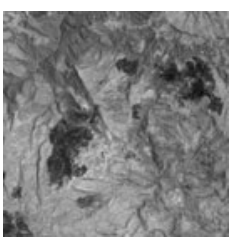

(b)

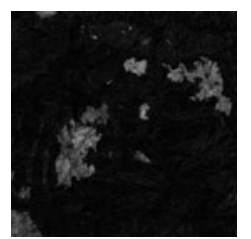

(c)

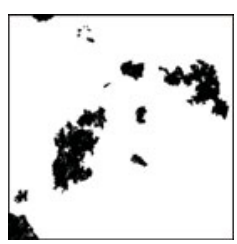

(d)

Fig. 1. Images of Mexico area. (a) Band 4 image acquired in April 2000, (b) band 4 image acquired in May 2002, (c) corresponding difference image, and (d) a reference map of the changed area.

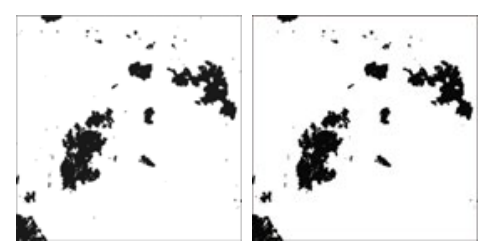

(a)

(b)

Fig. 2. Change detection maps obtained for Mexico data set: (a) using the unsupervised method based on optimal threshold, and (b) using the proposed semi-supervised technique based on optimal threshold (with $0.1 \%$ training pattern)

Table 1. Results and comparison

\begin{tabular}{|c|c|c|c|c|c|c|c|c|}
\hline $\begin{array}{l}\text { Techniques } \\
\text { used }\end{array}$ & Threshold & $\begin{array}{c}\text { Training } \\
\text { patterns } \\
\text { used }\end{array}$ & $\begin{array}{l}\text { Max/ } \\
\text { Min }\end{array}$ & $M A$ & $F A$ & $O E$ & Avg. $O E$ & Avg. $K M$ \\
\hline \multirow{2}{*}{$\begin{array}{l}\text { Unsupervised } \\
\text { (based } \\
\text { on MSOFM) }\end{array}$} & \multirow{2}{*}{$\begin{array}{c}0.216 \\
\text { (optimal) }\end{array}$} & \multirow[b]{2}{*}{-} & Min & 1366 & 1618 & 2984 & \multirow{2}{*}{$\begin{array}{c}2991.70 \\
(4.360046)\end{array}$} & \multirow{2}{*}{$\begin{array}{c}0.935532 \\
(0.000089)\end{array}$} \\
\hline & & & Max & 1366 & 1634 & 3000 & & \\
\hline \multirow{4}{*}{$\begin{array}{l}\begin{array}{l}\text { Supervised } \\
\text { (based } \\
\text { on MLP) }\end{array} \\
\end{array}$} & \multirow{4}{*}{ - } & \multirow[t]{2}{*}{$0.1 \%$} & Min & 1345 & 1429 & 2774 & \multirow{2}{*}{$\begin{array}{c}3086.40 \\
(186.277857)\end{array}$} & \multirow{2}{*}{\begin{tabular}{|c|}
0.927558 \\
$(0.004676)$
\end{tabular}} \\
\hline & & & Max & 2268 & 1139 & 3407 & & \\
\hline & & \multirow[t]{2}{*}{$0.5 \%$} & Min & 1269 & 1406 & 2675 & \multirow{2}{*}{$\begin{array}{c}2834.3 \\
(95.736148)\end{array}$} & \multirow{2}{*}{$\begin{array}{c}0.915087 \\
(0.001731)\end{array}$} \\
\hline & & & Max & 875 & 2192 & 3067 & & \\
\hline \multirow{4}{*}{\begin{tabular}{|l|} 
Proposed \\
semi-supervised \\
(based \\
on MSOFM) \\
\end{tabular}} & \multirow{4}{*}{$\begin{array}{c}0.216 \\
\text { (optimal) }\end{array}$} & \multirow[t]{2}{*}{$0.1 \%$} & Min & 1512 & 1218 & 2730 & \multirow{2}{*}{$\begin{array}{c}2741.40 \\
(6.666333)\end{array}$} & \multirow{2}{*}{$\begin{array}{c}0.940312 \\
(0.000141)\end{array}$} \\
\hline & & & Max & 1516 & 1239 & 2755 & & \\
\hline & & \multirow[t]{2}{*}{$0.5 \%$} & Min & 1470 & 1235 & 2705 & \multirow{2}{*}{$\begin{array}{c}2723.70 \\
(8.331266) \\
\end{array}$} & \multirow{2}{*}{\begin{tabular}{|c|}
0.940475 \\
$(0.000193)$ \\
\end{tabular}} \\
\hline & & & Max & 1502 & 1232 & 2734 & & \\
\hline
\end{tabular}

as compared to the corresponding unsupervised and semi-supervised approaches. This may be due to the unavailability of sufficient number of training samples which reflect the real life scenario. By comparing the results of the supervised and the proposed semi-supervised methods, it is seen that the maximum overall 
error (denoted by 'Max') obtained by the semi-supervised approach is less than that of the corresponding supervised method. In short, the results show that the proposed semi-supervised version has an edge over the unsupervised as well as the supervised techniques when a few labeled patterns are available.

\section{Conclusion}

In this paper, an attempt has been made to improve the performance of change detection of remotely sensed images under the scarcity of sufficient number of labeled patterns by exploring the self-organizing capacity of Kohonen's network integrated with semi-supervision. From the results, it has been found that the proposed algorithm is better suited for change detection where a small amount of labeled patterns is available.

Acknowledgments. The authors like to thank the Department of Science and Technology (DST), Government of India and University of Trento, Italy, the sponsors of the ITPAR program and Prof. L. Bruzzone, the Italian collaborator of this project, for providing the data.

\section{References}

1. Canty, M.J.: Image Analysis, Classification and Change Detection in Remote Sensing. CRC Press, Taylor \& Francis (2006)

2. Chapelle, O., Schölkopf, B., Zien, A.: Semi-supervised Learning. MIT Press, Cambridge (2006)

3. Congalton, R.G., Green, K.: Assessing the Accuracy of Remotely Sensed Data: Principles and Practices, 2nd edn. CRC Press, Taylor \& Francis Group (2009)

4. Ghosh, A., Pal, S.K.: Neural network, self-organization and object extraction. Pattern Recognition Letters 13, 387-397 (1992)

5. Ghosh, A., Mishra, N.S., Ghosh, S.: Fuzzy clustering algorithms for unsupervised change detection in remote sensing images. Information Sciences 181(4), 699-715 (2011)

6. Ghosh, S., Patra, S., Ghosh, A.: An unsupervised context-sensitive change detection technique based on modified self-organizing feature map neural network. International Journal of Approximate Reasoning 50(1), 37-50 (2009)

7. Kohonen, T.: Self-Organizing Maps, 2nd edn. Springer, Berlin (1997)

8. Patra, S., Ghosh, S., Ghosh, A.: Change detection of remote sensing images with semi-supervised multilayer perceptron. Fundamenta Informaticae 84, 429-442 (2008)

9. Singh, A.: Digital change detection techniques using remotely sensed data. International Journal of Remote Sensing 10(6), 989-1003 (1989) 